\title{
Photo cleavable thioacetal block copolymers for
}

\section{controlled release}

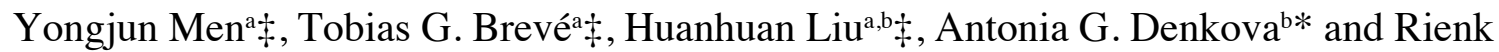

Eelkema $^{\text {a* }}$

aDepartment of Chemical Engineering, Delft University of Technology, van der Maasweg 9, 2629 HZ Delft, The Netherlands.

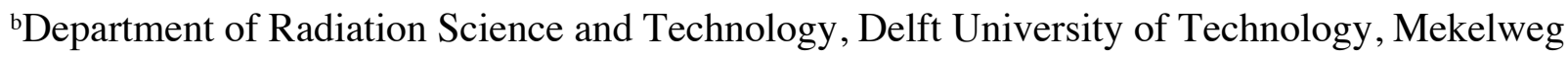
15, 2629 JB Delft, The Netherlands.

KEYWORDS: thioacetal, targeted chemotherapy, photocleavable polymer, block copolymer nanoparticles.

\begin{abstract}
We present a new light cleavable polymer containing $o$-nitrobenzene thioacetal groups in the main chain. By conjugation to a PEG block, we synthesized block copolymers capable of forming nanoparticles in aqueous solution. We studied drug encapsulation and release using the model drug Nile Red. Irradiation with UV-A light (365 nm) leads to efficient degradation of the polymers and associated burst release of the payload. Unlike other thioacetal and thioketal polymers, these polymers are stable to reactive oxygen species (ROS), preventing
\end{abstract}


non-triggered release. Moreover, the nanocarriers showed low cytotoxicity in cell viability experiments.

\section{INTRODUCTION}

Stimuli-responsive polymers are of great interest in nanomedicine since they allow to accurately release drugs at the diseased site, while minimizing side effects to healthy tissues. ${ }^{1-3}$ Such polymers have been used for drug release by encapsulating drugs or directly linking drugs to the polymer side chains. ${ }^{4-7}$ Changes of the polymer physical properties (e.g., the aggregation state) or its chemical structure can lead to drug release, in response to triggers emanating from the body (e.g., $\mathrm{pH}$, temperature, biomarkers, reactive oxygen species (ROS)) or from external sources (e.g., light, heat, magnetic fields, ultrasound) ${ }^{8-20}$ Light has multiple advantages for use as a trigger, including easy and local application, and fast response. These advantages have led to a great interest in photocleavable polymers, which offer the potential for spatiotemporal control over the cleavage of covalently linked prodrugs or the disassembly of block copolymer micelles or vesicles. ${ }^{21}$ Photolabile compounds, or photocages, include $o$-nitrobenzene $(o-\mathrm{NB})$, nitrobenzofuran, 6-bromo-7-hydroxycoumarin, quinolone, cyanine, and others..$^{22-25}$ Among these, $o$ NB equipped polymers have been widely investigated due to the versatility of $o$-NB monomers. ${ }^{26}$ $o$-NB based linkers and protecting groups undergo photocleavage upon UV-A irradiation (315$400 \mathrm{~nm}$ ), a process that takes place on a time scale of minutes to several hours depending on the light intensity used and the quantum yield of the light sensitive moiety. ${ }^{27}$

Three approaches have been reported to prepare $o$-NB based polymers.$^{28}$ In the first approach, a $o$-NB-functionalized alkene type monomer is polymerized via free radical polymerization. The length of the backbone of these $o$-NB-decorated polymers remains unchanged after cleaving the $o$-NB moieties upon UV irradiation. ${ }^{29}$ An advantage of this approach is the possibility to achieve 
a high degree of substitution by the $o$-NB moiety. On the other hand, this approach requires complicated monomer synthesis and shows slow drug release rates since the polymer backbone is not degraded, and many $o$-NB moieties must be removed to achieve the desired effect. The second approach is the synthesis of a block copolymer, in which an $o$-NB derivative is used as covalent photocleavable linker between the blocks. ${ }^{28,30}$ The third approach relies on condensation polymerization of $o$-NB derivatives to obtain polymers that include $o$-NB moieties in their backbone ${ }^{31}$ In this polymer type $o$-NB enables the cleavage of the polymer backbone upon UV irradiation, leading to a fast drug release, since a small fraction of cleaved bonds results in a large decrease in the polymer molecular weight. One example is polymerizing $o$ nitrobenzaldehyde (o-NBA) with monomers containing carboxylic acid and isocyanide via the Passerini reaction..$^{32,33}$

In 2010, Murthy et al. reported a thioketal-based polymer synthesized by condensation of low molecular weight thiols and ketones..$^{34}$ The thioketal bonds can be easily broken by reaction with ROS while they remain stable in harsh acid and alkaline conditions, providing a possibility for oral drug delivery. In another report, Choi et al. prepared a thioacetal o-NBA dual-arm photocage that enabled control of the simultaneous release of two payloads linked to a single unit, and the photocage showed a quantum yield of $0.2 .35,36$ Inspired by these reports we anticipated that a thioacetal polymer synthesized through condensation polymerization of $o$-NBA and a dithiol will have photoresponsive properties. ${ }^{34,37}$ Furthermore, any remaining thiol end groups can be easily conjugated to hydrophilic polymers through maleimide click chemistry, to prepare amphiphilic block copolymers that can assemble into nanoparticles and can be used as a photo-cleavable drug carrier (Figure 1). 
a)

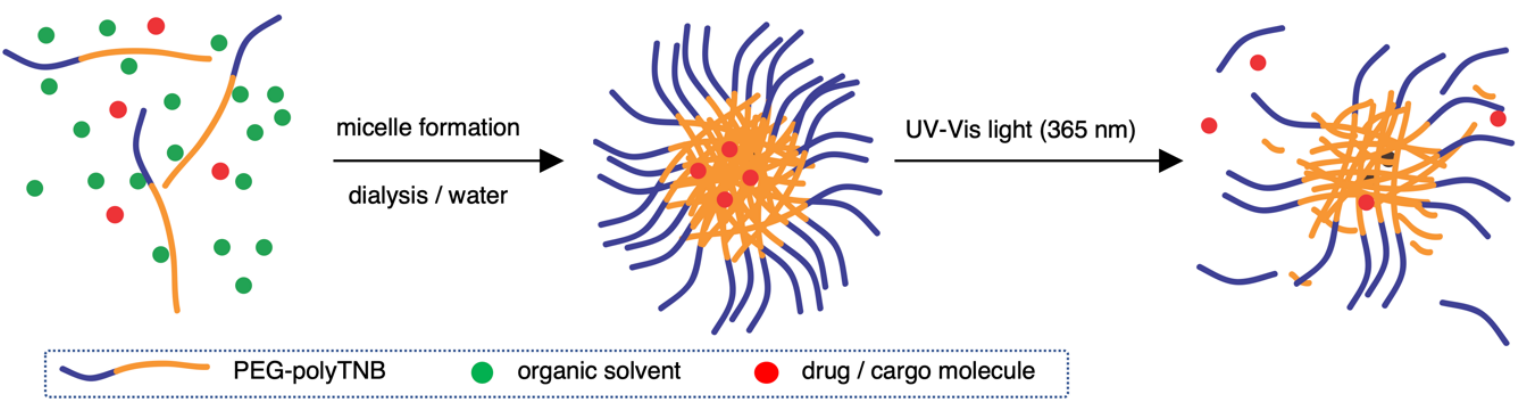

b)
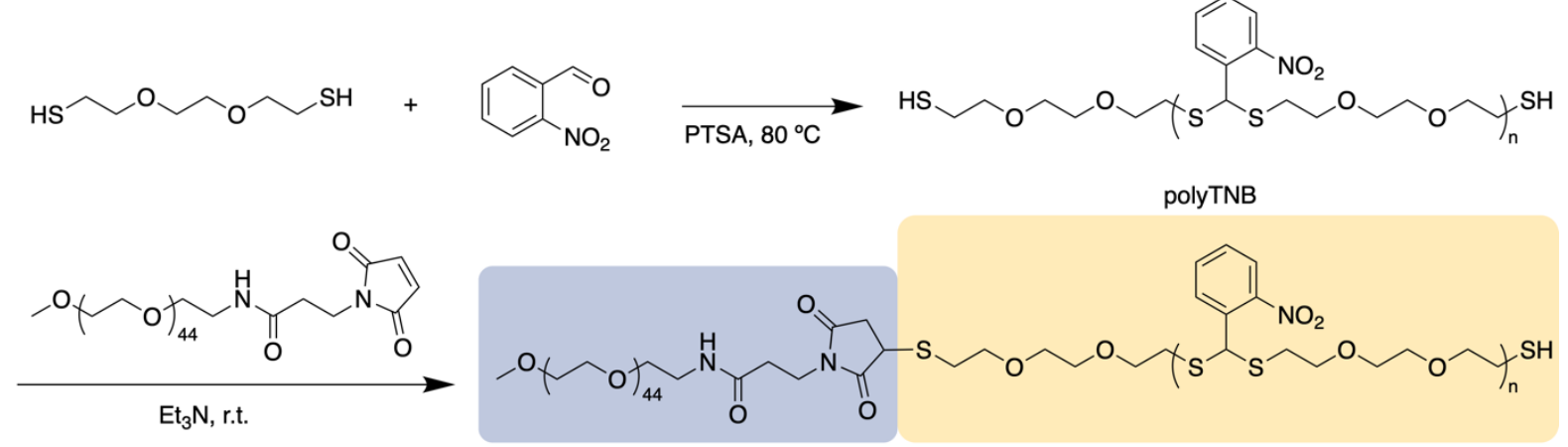

PEG-polyTNB

Figure 1. Scheme of PEG-polyTNB nanoparticle assembly and light triggered release. a) Block copolymer micelle formation and light-triggered drug release; b) Synthesis of PEG-polyTNB block copolymer from dithiol and $o$-nitrobenzaldehyde, followed by thia-Michael addition to PEG-maleimide. PTSA = p-toluene sulfonic acid.

\section{EXPERIMENTAL SECTION}

Materials All the chemicals were bought from Sigma-Aldrich and used without further purification. Benzyl mercaptan (99\%), o-nitrobenzaldehyde (98\%), p-toluenesulfonic acid (>98.5\%), methoxypolyethylene glycol maleimide (mPEG-maleimide, $\mathrm{M}_{\mathrm{w}}=2 \mathrm{kDa},>90 \%$ ), Nile Red (>98\%), Doxorubicin (98.0-102.0\%).

Synthesis of polyTNB. A mixture of 3,6-dioxa-1,8-octanedithiol (1.62 mL, $10 \mathrm{mmol}), o$ nitrobenzaldehyde $(1.51 \mathrm{~g}, 10 \mathrm{mmol})$, and $p$-toluenesulfonic acid $(1.7 \mathrm{mg}, 10 \mu \mathrm{mol})$ as a catalyst was stirred at $80^{\circ} \mathrm{C}$ under argon protection for 4 days. The reaction mixture was precipitated into 
diethyl ether 3 times and the obtained polymer was then dried under vacuum overnight giving polyTNB as a yellow sticky solid (3.29 g, $99 \%)$. The ${ }^{1} \mathrm{H}-\mathrm{NMR}$ spectrum is shown in Figure S2.

Synthesis of PEG-polyTNB. We dissolved polyTNB (250 mg, $52.4 \mu \mathrm{mol})$ in chloroform (8 $\mathrm{mL}$ ), and added mPEG-maleimide of $2 \mathrm{kDa}(105 \mathrm{mg}, 52.4 \mu \mathrm{mol})$ and $\mathrm{Et}_{3} \mathrm{~N}$ (1 drop). The reaction mixture was magnetically stirred for $24 \mathrm{~h}$ at room temperature and then concentrated in vacuo to give an oily product. Deionized water $(20 \mathrm{~mL})$ was added to the crude product and stirred for 30 min. The precipitate was removed, and the precipitate was further washed with methanol (150 mL) 3 times. The obtained gray sticky solid was vacuum dried for $48 \mathrm{~h}$. Yield: $320 \mathrm{mg}$, $90 \%$.

Preparation of Nile Red Loaded Nanoparticles. We used a solvent switching method for the preparation of empty (control) polymeric nanoparticles and cargo-loaded nanoparticles. A typical procedure is as follows: PEG-polyTNB (20 mg) was dissolved in a mixture of tetrahydrofuran (THF) and 1,4-dioxane (dioxane) ( $2 \mathrm{~mL}, 4: 1$ by volume) with Nile Red (10 $\mu \mathrm{L}, 20 \mathrm{mg} / \mathrm{mL}$ in DMSO) in a $15 \mathrm{~mL}$ capped vial with a magnetic stirrer. After the compounds were stirred for 30 min at room temperature, we added water at a rate of $1 \mathrm{~mL} / \mathrm{h}$ using a syringe pump. The needle from the syringe was inserted into the vial of which the cap was replaced with a rubber septum. 2 $\mathrm{mL}$ of water was pumped into the organic solution with vigorous stirring (900 rpm). After the water addition was finished, the suspension was transferred to a dialysis tube and dialysis against Milli-Q water 3 L for 48 hours with changing frequency of every 12 hours.

Encapsulation Efficiency. Encapsulation efficiency (EE\%) of dye-loaded nanoparticles was calculated by the following formula:

$\mathrm{EE}(\%)=($ weight of dye determined by fluorescence /weight of feeding dye $) \times 100 \%$

The amount of Nile Red was measured by the fluorescence intensity. We first draw a standard curve by using the fluorescence intensity of a series of variant concentrations ( 0.001 to 0.1 
$\mu \mathrm{g} / \mathrm{ml}$ ) of Nile Red in tetrahydrofuran (THF) (Ex: $527 \mathrm{~nm}$, Em: $604 \mathrm{~nm}$ ). To determine the total amount of Nile Red associated with the nanoparticles, the nanoparticle solution was centrifugated to remove the water. Then the aggregated nanoparticles were dissolved in THF, and measured their fluorescence intensity.

Photo-responsiveness and photo-cleavage reaction. One milliliter of the PEG-polyTNB micelle solution $(2.5 \mathrm{mg} / \mathrm{mL})$ was exposed to UV light $\left(365 \mathrm{~nm}, 90 \mathrm{~mW} \mathrm{~cm}{ }^{-2}\right)$ for $0-15 \mathrm{~min}$ in a QS cuvette. At a certain time, $10 \mu \mathrm{L}$ of the solution was taken out and diluted to 40 times in water for UV and DLS measurements. $20 \mu \mathrm{L}$ of the solution was mixed with $200 \mu \mathrm{L}$ THF for GPC measurement. NMR samples were freeze-dried under vacuum (0.01 bar) overnight and redissolved in $0.5 \mathrm{~mL} \mathrm{CDCl}{ }_{3}$.

ROS-responsiveness. Structural changes of polymers in response to treatment with ROS reagents $\left(\mathrm{H}_{2} \mathrm{O}_{2}\right.$ or $\left.\mathrm{KO}_{2}\right)$ were measured using ${ }^{1} \mathrm{H}-\mathrm{NMR}$ spectroscopy. To $1 \mathrm{~mL}$ of the polymeric micelle solution $\left(2.5 \mathrm{mg} / \mathrm{mL}\right.$ ) in PBS (pH 7.4) we added $\mathrm{H}_{2} \mathrm{O}_{2}(10 \mathrm{M})$ to form a $500 \mathrm{mM}$ solution or $\mathrm{KO}_{2}$ to form a $100 \mathrm{mM}$ solution. After incubation at $37{ }^{\circ} \mathrm{C}$ for $24 \mathrm{~h}$, the solution was lyophilized, and the product was studied by ${ }^{~} \mathrm{H}$ NMR.

Dye release behavior. We performed cargo release experiments of Nile Red loaded polymeric nanoparticles in neutral PBS ( $\mathrm{pH}$ 7.4). The Nile Red release behavior after UV365 light irradiation was investigated by fluorescence measurement. Specifically, $1 \mathrm{~mL}$ nanoparticles $(2.5$ $\mathrm{mg} / \mathrm{mL}$ ) was transferred into a dark QS cuvette. The fluorescence of the release solution at different time intervals was measured at $601 \mathrm{~nm}$ emission wavelengths by a microplate reader (excitation wavelength at $530 \mathrm{~nm}$ ).

Cytotoxicity of PEG-polyTNB nanoparticles. HeLa cells were kept in DMEM culture medium (Dulbecco's Modified Eagle Medium, Biowest) supplemented with $10 \% \mathrm{~mL}$ fetal 
bovine serum (Gibco, Life Technologies) and 1\% Penicillin/Streptomycin (100x, Biowest) under humidified normoxic conditions $\left(95 \%\right.$ air, $\left.5 \% \mathrm{CO}_{2}\right)$ at $37{ }^{\circ} \mathrm{C}$. For evaluating the in vitro cytotoxicity of the PEG-polyTNB nanoparticles, $2000 \mathrm{HeLa}$ cells (suspended in $200 \mu \mathrm{L}$ cell culture medium) were seeded in 96-well plates and incubated for three days. $10 \mu \mathrm{L}$ of PEGpolyTNB nanoparticles was added to each well, with the final micelle concentration of 0.119 $\mathrm{mg} / \mathrm{mL}$. After being incubated for another $24 \mathrm{~h}$, the old culture medium was removed, the cells were washed with PBS twice and $200 \mu \mathrm{L}$ of fresh culture medium was added to Hela cells. Another 3 days has been given for the cell to growth before the WST-8 assay (Cell Counting Kit8, Dojindo Laboratories, Tebu-Bio). For the test, $10 \mu \mathrm{L}$ of CCK-8 reagent was given to each well and incubated for $3 \mathrm{~h}$, then the absorptance at $450 \mathrm{~nm}$ was measured by a microplate scanning spectrophotometer (PowerWave XSTM, Bio-Tek). The surviving fraction (SF) of the Hela Cells was calculated using the following equation:

$$
\mathrm{SF}=\frac{A b s(450)_{\text {sample }}-A b s(450)_{\text {blank }}}{A b s(450)_{\text {controll }}-A b s(450)_{\text {blank }}}
$$

Abs(450)sample is the absorptance at $450 \mathrm{~nm}$ for cells with PEG-polyTNB nanoparticles; Abs(450)control is the absorptance at $450 \mathrm{~nm}$ for cells with $10 \mu \mathrm{L}$ of culture medium;

Abs(450) blank is the absorptance at $450 \mathrm{~nm}$ for vials without addition of CCK-8 reagent.

\section{RESULTS AND DISCUSSION}

\section{Synthesis and characterization of polyTNB}

The synthesis scheme of PEG-polyTNB block copolymer is shown in Figure 1b. 3,6-Dioxa1,8-octanedithiol and o-nitrobenzaldehyde were polymerized to polyTNB in a bulk polymerization using $p$-toluenesulfonic acid (PTSA) as catalyst. The obtained polyTNB shows highly elastic (low glass transition temperature, $\mathrm{T}_{\mathrm{g}}=-17{ }^{\circ} \mathrm{C}$, Figure $\mathrm{S} 1$ ) behavior due to the 
ethylene glycol segments in the polymer chain. A peak at $5.89 \mathrm{ppm}$ that belongs to the proton in the thioacetal group could be observed in ${ }^{1} \mathrm{H}-\mathrm{NMR}$ (Figure S2), while the starting material aldehyde peak of $o$-NBA is absent. The molecular weight $\left(\mathrm{M}_{\mathrm{n}}\right)$ of polyTNB is $8.0 \mathrm{~kg} / \mathrm{mol}(\bigoplus$ $=2.92$, Figure $2 \mathrm{~b}$ ) determined by gel permeation chromatography (GPC). The broad molecular weight distribution results from the condensation polymerization method. The dried polyTNB was then reacted with an equimolar amount of methoxy-PEG-maleimide assisted by a catalytic amount of triethylamine (TEA) at room temperature for $24 \mathrm{~h}$ to form the PEG-polyTNB block copolymer. All peaks in the ${ }^{1} \mathrm{H}-\mathrm{NMR}$ spectrum (Figure 2a) correspond to PEG-polyTNB, with integration of the aliphatic peaks suggesting that the major product is the mono-PEGylated polyTNB. The molecular weight of PEG-polyTNB as measured by GPC $\left(\mathrm{M}_{\mathrm{n}}=8.6 \mathrm{~kg} / \mathrm{mol}, \boxplus\right.$ $=3.30$, Figure $2 \mathrm{~b}$ ) is somewhat lower than the expected $10.0 \mathrm{~kg} / \mathrm{mol}$ (polyTNB $8.0 \mathrm{~kg} / \mathrm{mol}+$ methoxy-PEG-maleimide $2.0 \mathrm{~kg} / \mathrm{mol}$ ), likely due to two reasons. First, the radius of gyration of PEG-polyTNB in THF (GPC mobile phase) is different as that of single PEG and polyTNB. Second, a small percentage of polyTNB remained unreacted or conjugated to two PEG chains (PEG-polyTNB-PEG), resulting in a broader dispersity.
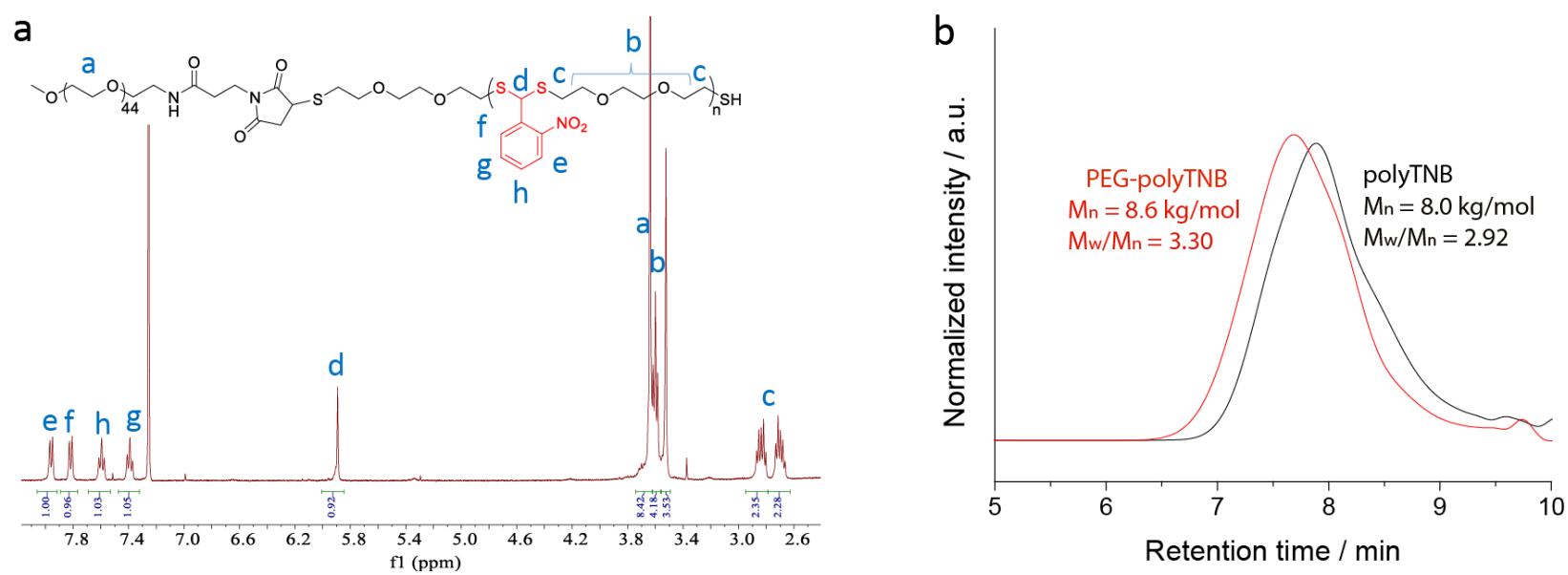

Figure 2. a) ${ }^{1} \mathrm{H}-\mathrm{NMR}$ spectrum of PEG-polyTNB in $\mathrm{CDCl}_{3}$; b) Molecular weight and dispersity of polyTNB and PEG-polyTNB measured by GPC. 


\section{Characterization of PEG-polyTNB nanoparticles}

For the preparation of PEG-polyTNB nanoparticles, we used a solvent switching method. PEG-polyTNB (10 mg) was dissolved in THF/dioxane (vol/vol $1 \mathrm{~mL})$, followed by the slow addition $(2 \mathrm{~mL} / \mathrm{h})$ of $1 \mathrm{~mL} \mathrm{H}_{2} \mathrm{O}$. The turbid transition (i.e. critical aggregation concentration) occurred at $24-25$ vol\% $\mathrm{H}_{2} \mathrm{O}$ content. The organic solvents were removed by dialyzing the suspension against Milli-Q water for $48 \mathrm{~h}$. The mean size of the obtained nanoparticles was 159 $\mathrm{nm}(\mathrm{PDI}=0.082)$ as measured by DLS. The TEM analysis shows that the nanoparticles appear as nearly spherical shapes, with smaller size $(\sim 100 \mathrm{~nm})$ than DLS due to the drying effect (Figure 3e).

\section{Light degradation of PEG-polyTNB nanoparticles}
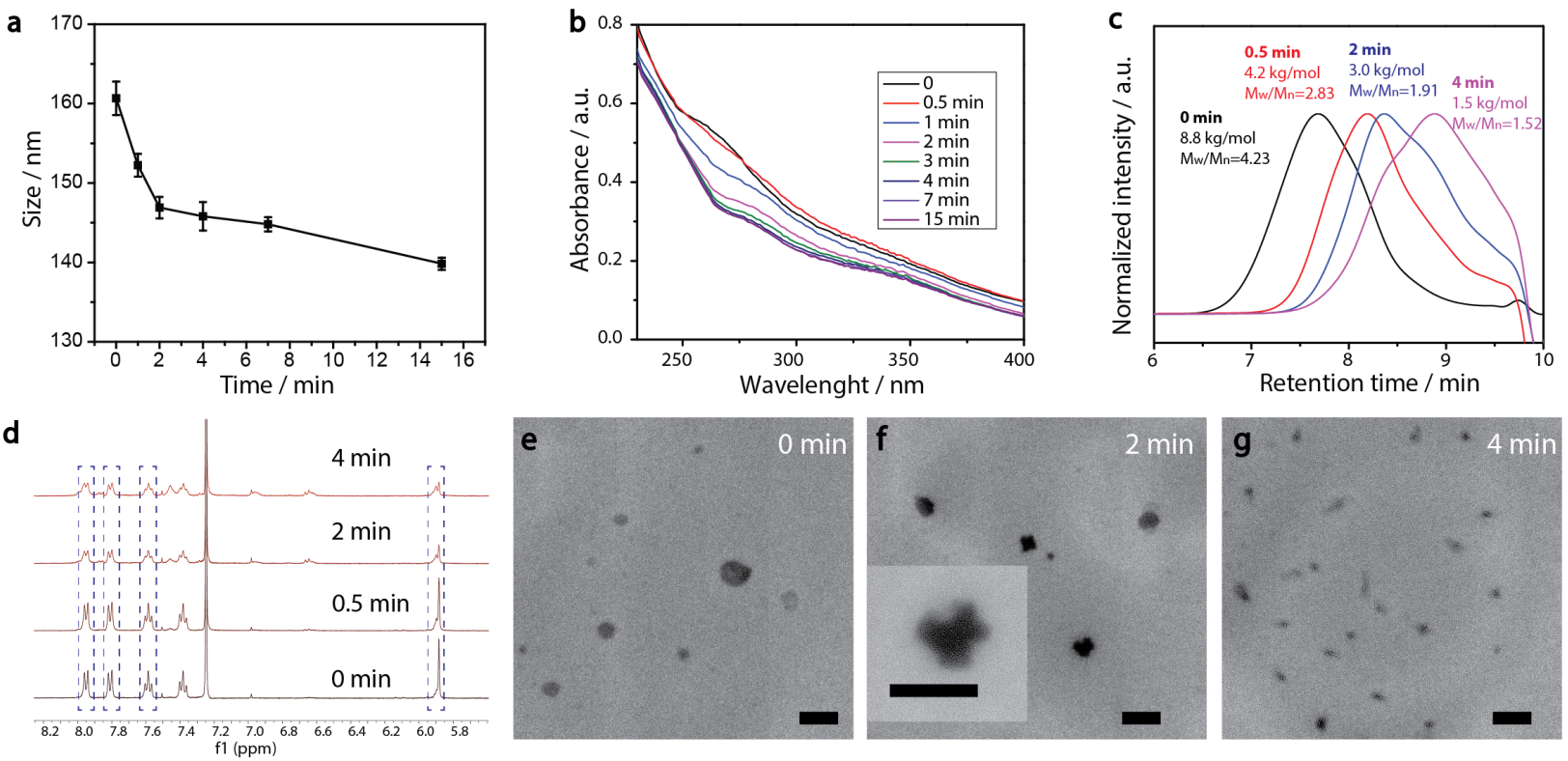

Figure 3. PEG-polyTNB nanoparticle degradation depending on the duration of UV-A (365nm)

exposure. a) Size changes measured by DLS; error bars represent standard deviation based on three measurements. b) UV-vis absorption spectra changes; c) molecular weight and distribution change measured by GPC using polystyrene standards for reference; d) molecular structure 
changes measured by ${ }^{1} \mathrm{H}-\mathrm{NMR}$ (PEG unit was chosen as calibration standard due to no signal change during the reaction); e-g) morphology changes measured by TEM, scale bar $200 \mathrm{~nm}$.

PEG-polyTNB nanoparticles are expected to decompose upon UV-A light (365 nm) irradiation resulting from the photolability of the $o$-nitrobenzyl unit. To examine the light-triggered degradation, we exposed $1 \mathrm{~mL}$ of PEG-polyTNB nanoparticles solution $(2.5 \mathrm{mg} / \mathrm{mL})$ under 365 $\mathrm{nm}$ light with an intensity of $90 \mathrm{~mW} \cdot \mathrm{cm}^{-2}$, which is lower than the Maximum Permissible Exposure $\left(200 \mathrm{~mW} \cdot \mathrm{cm}^{-2}\right)$. DLS results show the nanoparticle size decreased from $159 \mathrm{~nm}$ to 146 $\mathrm{nm}$ in the first $2 \mathrm{~min}$, and slowly reduced to $140 \mathrm{~nm}$ in the following $13 \mathrm{~min}$, indicating that the decomposition reaction proceeded quickly in the first $2 \mathrm{~min}$ which is consistent with the results of the UV experiment (Figure 2b). After being irradiated for $15 \mathrm{~min}$, no precipitation observed from the nanoparticle suspension, but shows a slightly decrease of zeta potential that changed from - $41.8 \mathrm{mV}$ (before irradiation) to $-34.1 \mathrm{mV}$ (UV $0.5 \mathrm{~min}$ ) and $-32.6 \mathrm{mV}$ (UV $4 \mathrm{~min}$ ) (Figure S3). The morphology of nanoparticles changed from a spherical shape to irregular shapes within 2 min of irradiation as was observed from TEM images (Figure 2e-g). These shape changes might be caused by polymer chain rearrangement induced by backbone breaks. To understand the chemical change of the polymer backbone at the molecular level, we analyzed the nanoparticles by NMR and GPC after freeze-drying of the nanoparticles. ${ }^{1} \mathrm{H}-\mathrm{NMR}$ (Figure 3d) shows how the peak at $\delta=7.59 \mathrm{ppm}$, belonging to an aromatic proton in the nitrobenzyl group (proton g, Figure 1a) decreased on average $12 \%, 34 \%$, and $50 \%$ after UVA irradiation for 0.5, 2, and $4 \mathrm{~min}$, respectively (Figure S4). Since a small fraction of bond breaking of the polymer will result in a large reduction of the polymer average molecular weight, by only 0.5 min UVA irradiation the average $\mathrm{M}_{\mathrm{n}}$ of PEG-polyTNB drops from $8.8 \mathrm{~kg} / \mathrm{mol}(\bigoplus=4.23)$ to $4.2 \mathrm{~kg} / \mathrm{mol}$ ( 
$=2.83$ ) and further to $3.0 \mathrm{~kg} / \mathrm{mol}(\bigoplus=1.91$, UV $2 \mathrm{~min})$ and $2.5 \mathrm{~kg} / \mathrm{mol}(\bigoplus=1.52$, UV $4 \mathrm{~min})$, as shown in Figure 3c. We observed that the molecular weight of PEG-polyTNB is different before $(8.6 \mathrm{~kg} / \mathrm{mol}, Ð=3.30)$ and after micellization $(8.8 \mathrm{~kg} / \mathrm{mol}, Ð=4.23)$. This effect may be caused by unreacted polymer chain end thiol groups forming disulfide bonds that increased the polymer molecular weight and distribution during the nanoparticle formation and lyophilization processes. After $4 \mathrm{~min}$ irradiation, the molecular weight remained above $2000 \mathrm{~g} / \mathrm{mol}$ as the PEG block ( 2000 g/mol) is non-photo degradable, indicating that the mixture obtained after irradiation mainly contains short oligomers of polyTNB and low molecular weight PEGpolyTNB. These shortened PEG-polyTNB fragments may still play a role as the stabilizer of nanoparticles, but the small fraction of PEG that completely cleaved from the hydrophobic polyTNB would release into solution, which explains the observed zeta potential decrease.

In an attempt to shed light on the mechanism of the photochemical thioacetal cleavage reaction, we synthesized a small molecule, ((2-nitrophenyl)methylene)bis(octylsulfane) (NBA, Figure 4 and S5), an analog of the TNB repeat unit. NBA contains the same thioacetal group as TNB, but has alkyl substituents on sulfur instead of the glycol units in TNB, to simplify the structure. Irradiation of NBA with UV-A (24h) led to complete conversion of the NBA structure, as shown by the disappearance of the $5.49 \mathrm{ppm}$ thioacetal proton peak in the ${ }^{1} \mathrm{H}-\mathrm{NMR}$ spectrum (Figure S6). After purification of the product mixture, we characterized the main product by ${ }^{1} \mathrm{H}-$ NMR, FTIR and mass spectrometry (Figures S7, S8, S10). Irradiation of $o$-nitrobenzyl derivatives will often lead to $o$-nitrosobenzyl products.$^{28}$ In our case, however, the analytical data of the product did not align with the $o$-nitroso product. Instead, we found evidence suggesting the formation of an $o$-amino benzoic acid thioester (Figure 4). FTIR (Figure S10) showed strong bands at 3475 and $3365 \mathrm{~cm}^{-1}$, suggesting the existence of a primary amine; mass spectrometry 
showed a main signal at $\mathrm{m} / \mathrm{z} 266,16$ Da less than the benzisoxazole intermediate, indicating a dehydration process may have occurred. Having an amine product instead of the nitroso means a formal reduction. Building on a reported mechanism of photolysis of 1-(2-nitrophenyl) ethyl phosphates in the presence of thiol, ${ }^{38}$ we propose a mechanism as shown in Figure 4 . The mechanism proceeds to first give the common nitroso product, which is then further reduced to give the reported benzisoxazole intermediate and finally the amine product. In ${ }^{1} \mathrm{H}-\mathrm{NMR}$ and MS measurements we observed the formation of dioctyl disulfide, the typical oxidation product of octyl thiol (Figures S6, S9). The large amount of dioctyl disulfide suggests that octyl thiol plays a role in the process of nitroso reduction, although this is by no means a clean process and other mechanisms may also be at play. In the polymer irradiation experiments, we do not observe the formation of high molecular weight disulfide polymers. This result suggests that the polymer breakdown either follows a partially different mechanism or that the formed thiols may be converted to low molecular weight cyclic or linear disulfide fragments. 


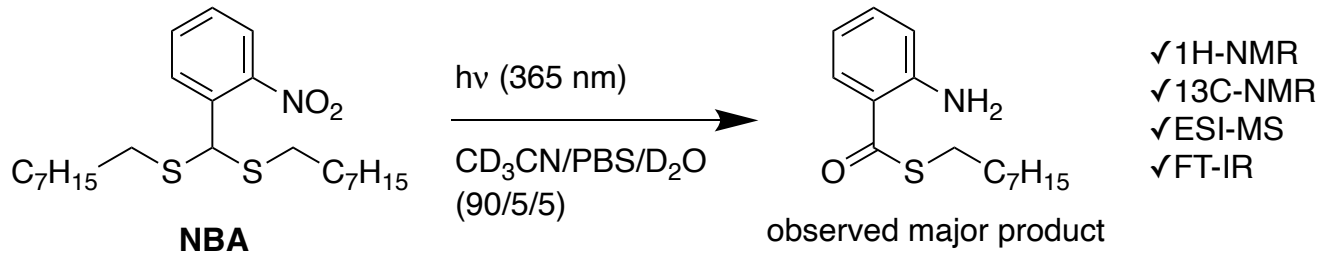

proposed mechanism:

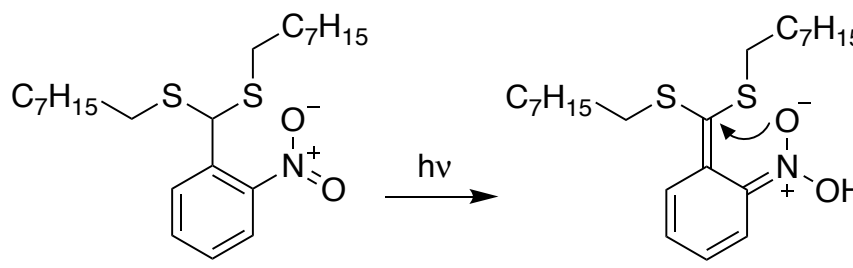<smiles>CCCCCCCCS[C@]1(SCCC)CCN(O)c2ccccc21</smiles>

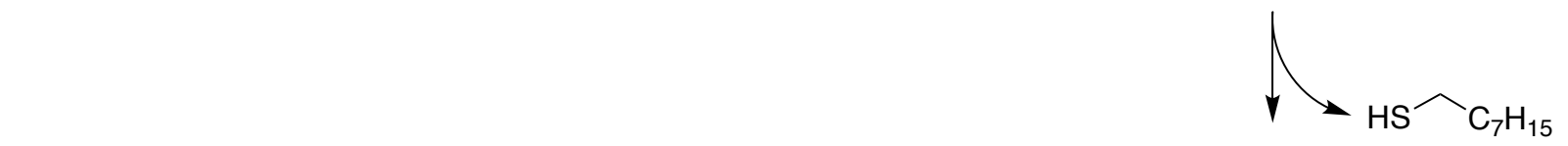<smiles>CCSSC[C@H]1CC[C@H]2C[C@H](C1)C2</smiles>

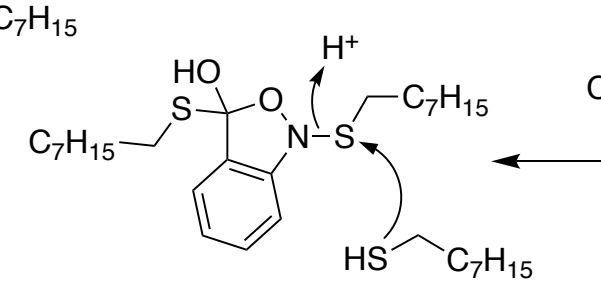

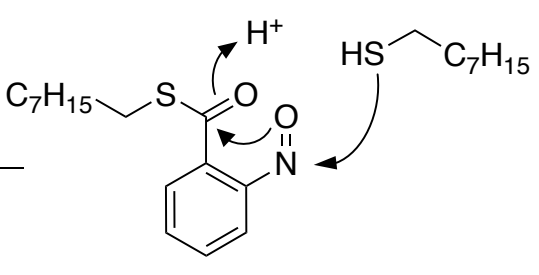<smiles>[3H][V]</smiles>

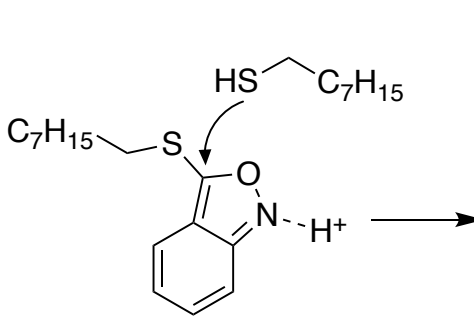

benzisoxazole intermediate

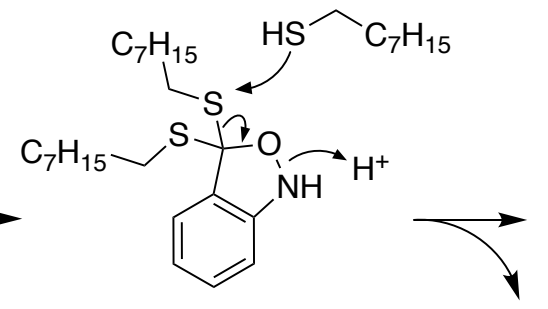

$\mathrm{C}_{7} \mathrm{H}_{15} \smile \mathrm{S}_{\mathrm{S}} \sim_{\mathrm{C}_{7} \mathrm{H}_{15}}$

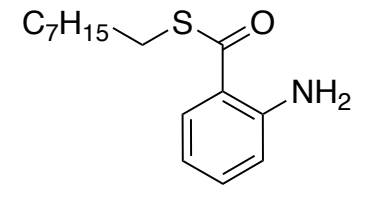

thioester amine isolated

Figure 4. The photochemical cleavage of NBA, and a proposed reaction mechanism for the formation of the observed thioester amine product.

\section{ROS-response of PEG-polyTNB nanoparticles}


As thioketal and thioacetal groups have been reported as being labile to reactive oxygen species (ROS), we also assessed the sensitivity of the nitrobenzylthioacetal derived polymers to some ROS types. We incubated PEG-polyTNB nanoparticles in PBS solution with $\mathrm{H}_{2} \mathrm{O}_{2}(500$ $\mathrm{mM})$ or $\mathrm{KO}_{2}(100 \mathrm{mM})$ at $37^{\circ} \mathrm{C}$ for $24 \mathrm{~h}$, and analyzed the resulting mixtures after lyophilization by ${ }^{1} \mathrm{H}-\mathrm{NMR}$. The results (Figure S11) show that the thioacetal structure was changed (new peaks appeared at $\delta=7.67,7.52$ and $6.01 \mathrm{ppm}$ ) in $500 \mathrm{mM} \mathrm{H}_{2} \mathrm{O}_{2}$ but surprisingly remained stable in $100 \mathrm{mM} \mathrm{KO} \mathrm{KO}_{2}$ and $\mathrm{H}_{2} \mathrm{O}_{2}$. These results demonstrated that PEG-polyTNB could respond to concentrated ROS, but proved much more stable than the often reported dimethylene thioketal based polymers that disintegrate when exposed to $\mathrm{H}_{2} \mathrm{O}_{2}$ and $\mathrm{KO}_{2}$ at a concentration of $10 \mathrm{mM} .^{11}$, 34,39 This substantial difference might be due to the electron withdrawing property of the nitro group that elevated the oxidation potential of the thioacetal compound.

\section{Light-triggered cargo release from PEG-polyTNB nanoparticles}

To evaluate the PEG-polyTNB nanoparticles as responsive carriers for drugs and other bioactive molecules, we first measured encapsulation and release of Nile Red as a hydrophobic drug model. Nile Red shows almost no fluorescence in water but can be intensely fluorescent in an apolar environment. The encapsulation efficiency (EE) of Nile Red in PEG-polyTNB nanoparticles was $42.6 \%$, demonstrating a high hydrophobic cargo loading capacity of PEGpolyTNB nanoparticles. The dye release experiment was performed in PBS under UV-A $(365 \mathrm{~nm})$ at various time points (Figure 5). The release rate decreased with irradiation time. When the nanoparticles were radiated for $0.5,2$, and $4 \mathrm{~min}, 15.4 \%, 37.5 \%$, and $52.9 \%$ Nile Red were released, respectively. After 10 min continues irradiation, the Nile Red release process almost reached equilibrium at about $70-80 \%$. And no release was observed without irradiation in the same period. 

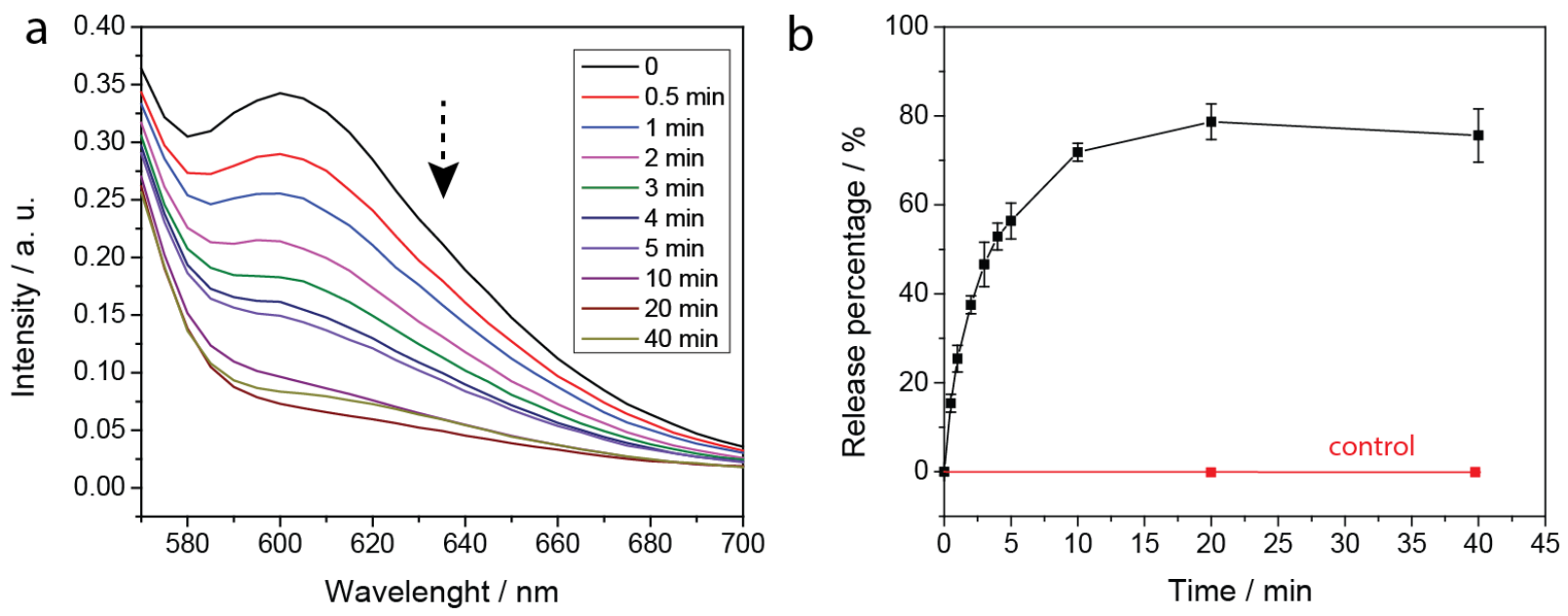

Figure 5. Nile Red release from NR-loaded nanoparticles in PBS under UV-A (365 nm) measured by fluorescence. a) Nile Red fluorescence intensity after irradiation; b) percentage of released Nile Red with increasing irradiation time; error bars represent the standard deviation of three measurements.

\section{Light-controlled cytotoxicity of PEG-polyTNB nanoparticles}

The cytocompatibility of the empty nanoparticles was evaluated using a human cervix carcinoma cell line (HeLa). The survival fraction of HeLa cells was higher than 90\% after incubation for $24 \mathrm{~h}$ at a micelle concentration of $0.119 \mu \mathrm{g} / \mathrm{mL}$ (Figure 6). These results indicated that the polymeric nanoparticles are biocompatible and show low cytotoxicity. But after intense UV-A $\left(365 \mathrm{~nm}, 90 \mathrm{~mW} \cdot \mathrm{cm}^{-2}\right)$ irradiation for $30 \mathrm{~s}$, only $60 \%$ of untreated cells was alive. Interestingly, cell viability after irradiation increased by about $20 \%$ when incubated with PEGpolyTNB nanoparticles. This effect may be related to the release of thiols into the cell environment upon photodegradation of polyTNB, where these thiols protect the cells against ultraviolet light by acting as an antioxidant. ${ }^{40}$ 


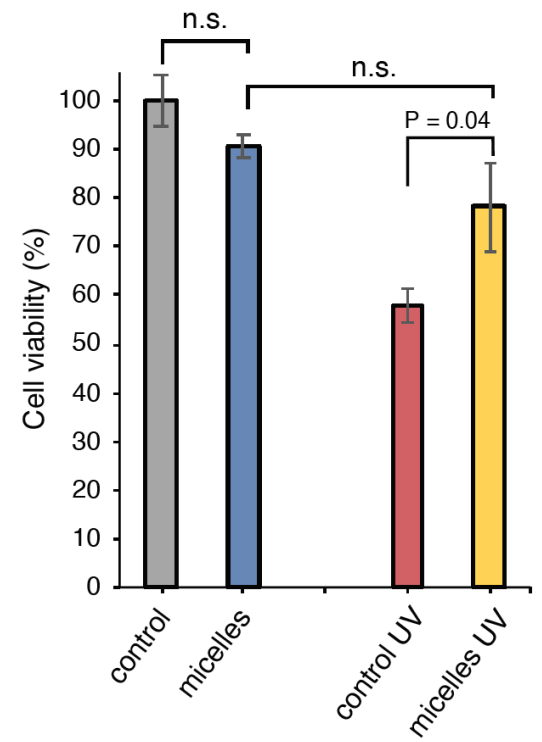

Figure 6. Viability of HeLa cells after $24 \mathrm{~h}$ with PEG-polyTNB nanoparticles and/or $30 \mathrm{~s}$ exposure to UV365 $\left(90 \mathrm{~mW} \cdot \mathrm{cm}^{-2}\right)$. A control without treatment was considered $100 \%$ viability. n.s. = not significant; error bars are standard deviation $(\mathrm{N}=3)$.

\section{CONCLUSION}

In conclusion, we report a facile method for the synthesis of photo-cleavable polymers having a photoresponsive moiety incorporated in the polymer backbone based on thioacetal formation with $o$-nitrobenzaldehyde. The thiol end group of polyTNB can be clicked to PEG, to form an amphiphilic block copolymer that can be employed for the assembly of nanoparticles. The nanoparticles show fast release of drug model Nile Red (>50 \%) in 5 min when exposed to UVA light. Moreover, the polyTNB nanoparticles, unlike other reported polymers containing thioacetal bonds, are resistant to high concentrations of ROS. For example, the structure kept stable over $24 \mathrm{~h}$ in environments containing up to $100 \mathrm{mM} \mathrm{KO} \mathrm{K}_{2}$ or $\mathrm{H}_{2} \mathrm{O}_{2}$. Lastly, we demonstrated the PEG-polyTNB nanoparticles are biocompatible. We expect that this easily synthesized photocleavable polymer can be used as a drug carrier for photodynamic therapy by combination with other energy up-conversion nanoparticles. ${ }^{41}$ 


\section{ASSOCIATED CONTENT}

\section{Supporting Information.}

The Supporting Information is available free of charge at

\section{AUTHOR INFORMATION}

\section{Corresponding Author}

Antonia G. Denkova

Email: A.G.Denkova@tudelft.nl

Rienk Eelkema

Email: r.eelkema@tudelft.nl

\section{Author Contributions}

$\$$ These authors contributed equally.

\section{Funding Sources}

European Research Council (ERC consolidator grant 726381)

Netherlands Organization for Scientific Research (TTW-OTP project 13306)

\section{ACKNOWLEDGMENT}


Generous funding by the Netherlands Organization for Scientific Research (TTW-OTP project 13306) and the European Research Council (ERC consolidator grant 726381) is acknowledged.

\section{REFERENCES}

1. De las Heras Alarcón, C.; Pennadam, S.; Alexander, C., Stimuli responsive polymers for biomedical applications. Chem. Soc. Rev. 2005, 34 (3), 276-285.

2. Jeong, B.; Gutowska, A., Lessons from nature: stimuli-responsive polymers and their biomedical applications. Trends Biotechnol. 2002, 20 (7), 305-311.

3. Denkova, A. G.; Liu, H.; Men, Y.; Eelkema, R., Enhanced Cancer Therapy by Combining Radiation and Chemical Effects Mediated by Nanocarriers. Adv. Ther. 2020, 3 (3), 1900177.

4. Wang, J.; Zhang, Y.; Archibong, E.; Ligler, F. S.; Gu, Z., Leveraging $\mathrm{H}_{2} \mathrm{O}_{2}$ Levels for Biomedical Applications. Adv. Biosyst. 2017, 1 (9), 1700084.

5. $\quad$ Liang, J.; Liu, B., ROS-responsive drug delivery systems. Bioengineering \& Transla. Med. 2016, 1 (3), 239251.

6. $\quad$ Deepagan, V. G.; Kwon, S.; You, D. G.; Nguyen, V. Q.; Um, W.; Ko, H.; Lee, H.; Jo, D.-G.; Kang, Y. M.; Park, J. H., In situ diselenide-crosslinked polymeric micelles for ROS-mediated anticancer drug delivery. Biomaterials 2016, 103, 56-66.

7. Ge, J.; Zhang, Q.; Zeng, J.; Gu, Z.; Gao, M., Radiolabeling nanomaterials for multimodality imaging: New insights into nuclear medicine and cancer diagnosis. Biomaterials 2020, 228, 119553.

8. Yang, B.; Chen, Y.; Shi, J., Reactive Oxygen Species (ROS)-Based Nanomedicine. Chem. Rev. 2019, 119 (8), 4881-4985.

9. DeRosa, M. C.; Crutchley, R. J., Photosensitized singlet oxygen and its applications. Coord. Chem. Rev. 2002, 233-234, 351-371.

10. Seah, G. L.; Yu, J. H.; Yang, M. Y.; Kim, W. J.; Kim, J.-H.; Park, K.; Cho, J.-W.; Kim, J. S.; Nam, Y. S., Lowpower and low-drug-dose photodynamic chemotherapy via the breakdown of tumor-targeted micelles by reactive oxygen species. J. Control. Release 2018, 286, 240-253.

11. Kim, J. S.; Jo, S. D.; Seah, G. L.; Kim, I.; Nam, Y. S., ROS-induced biodegradable polythioketal nanoparticles for intracellular delivery of anti-cancer therapeutics. J. Ind. Eng. Chem. 2015, 21, 1137-1142.

12. Song, C.-C.; Du, F.-S.; Li, Z.-C., Oxidation-responsive polymers for biomedical applications. J. Mater. Chem. B 2014, 2 (22), 3413-3426.

13. Xing, R.; Liu, K.; Jiao, T.; Zhang, N.; Ma, K.; Zhang, R.; Zou, Q.; Ma, G.; Yan, X., An Injectable SelfAssembling Collagen-Gold Hybrid Hydrogel for Combinatorial Antitumor Photothermal/Photodynamic Therapy. Adv. Mater. 2016, 28 (19), 3669-3676.

14. Zou, Q.; Zhang, L.; Yan, X.; Wang, A.; Ma, G.; Li, J.; Möhwald, H.; Mann, S., Multifunctional Porous Microspheres Based on Peptide-Porphyrin Hierarchical Co-Assembly. Angew. Chem. Int. Ed. 2014, 53 (9), 23662370 .

15. Zhao, F.; Shen, G.; Chen, C.; Xing, R.; Zou, Q.; Ma, G.; Yan, X., Nanoengineering of Stimuli-Responsive Protein-Based Biomimetic Protocells as Versatile Drug Delivery Tools. Chem. Eur. 2014, 20 (23), 6880-6887.

16. Fan, B.; Men, Y.; van Rossum, S. A.; Li, G.; Eelkema, R., A Fuel - Driven Chemical Reaction Network Based on Conjugate Addition and Elimination Chemistry. ChemSystemsChem 2020, 2 (1), e1900028.

17. Wu, S.; Zhang, Q.; Deng, Y.; Li, X.; Luo, Z.; Zheng, B.; Dong, S., Assembly Pattern of Supramolecular Hydrogel Induced by Lower Critical Solution Temperature Behavior of Low-Molecular-Weight Gelator. J. Am. Chem. Soc. 2019, 142 (1), 448-455.

18. Kohno, Y.; Saita, S.; Men, Y.; Yuan, J.; Ohno, H., Thermoresponsive polyelectrolytes derived from ionic liquids. Polym. Chem. 2015, 6 (12), 2163-2178. 
19. Men, Y.; Li, X.-H.; Antonietti, M.; Yuan, J., Poly (tetrabutylphosphonium 4-styrenesulfonate): a poly (ionic liquid) stabilizer for graphene being multi-responsive. Polym. Chem. 2012, 3 (4), 871-873.

20. Liu, B.; Thayumanavan, S., Mechanistic Investigation on Oxidative Degradation of ROS-Responsive Thioacetal/Thioketal Moieties and Their Implications. Cell Reports Physical Science 2020, 1 (12), 100271.

21. Nishiyama, N.; Morimoto, Y.; Jang, W.-D.; Kataoka, K., Design and development of dendrimer photosensitizer-incorporated polymeric micelles for enhanced photodynamic therapy. Advanced Drug Delivery Reviews 2009, 61 (4), 327-338.

22. Yamaguchi, T.; Takami, S.; Irie, M., Photochromic properties of 1, 2-bis (6-substitute-2-methyl-1benzofuran-3-yl) ethene derivatives. Journal of Photochemistry and Photobiology A: Chemistry 2008, 193 (2-3), 146-152.

23. Cabane, E.; Malinova, V.; Meier, W., Synthesis of photocleavable amphiphilic block copolymers: toward the design of photosensitive nanocarriers. Macromolecular chemistry and physics 2010, 211 (17), 1847-1856.

24. Furuta, T.; Wang, S. S.-H.; Dantzker, J. L.; Dore, T. M.; Bybee, W. J.; Callaway, E. M.; Denk, W.; Tsien, R. Y., Brominated 7-hydroxycoumarin-4-ylmethyls: photolabile protecting groups with biologically useful cross-sections for two photon photolysis. PNAS 1999, 96 (4), 1193-1200.

25. Zhao, Y., Light-Responsive Block Copolymer Micelles. Macromolecules 2012, 45 (9), 3647-3657.

26. Bagheri, A.; Yeow, J.; Arandiyan, H.; Xu, J.; Boyer, C.; Lim, M., Polymerization of a Photocleavable Monomer Using Visible Light. Macromol. Rapid Commun. 2016, 37 (11), 905-910.

27. Jiang, J.; Tong, X.; Zhao, Y., A new design for light-breakable polymer micelles. J. Am. Chem. Soc. 2005, 127 (23), 8290-8291.

28. Zhao, H.; Sterner, E. S.; Coughlin, E. B.; Theato, P., o-Nitrobenzyl alcohol derivatives: opportunities in polymer and materials science. Macromolecules 2012, 45 (4), 1723-1736.

29. Jiang, J.; Tong, X.; Morris, D.; Zhao, Y., Toward photocontrolled release using light-dissociable block copolymer micelles. Macromolecules 2006, 39 (13), 4633-4640.

30. Kang, M.; Moon, B., Synthesis of Photocleavable Poly(styrene-block-ethylene oxide) and Its Self-Assembly into Nanoporous Thin Films. Macromolecules 2009, 42 (1), 455-458.

31. Zhao, H.; Gu, W.; Sterner, E.; Russell, T. P.; Coughlin, E. B.; Theato, P., Highly Ordered Nanoporous Thin Films from Photocleavable Block Copolymers. Macromolecules 2011, 44 (16), 6433-6440.

32. Li, L.; Lv, A.; Deng, X.-X.; Du, F.-S.; Li, Z.-C., Facile synthesis of photo-cleavable polymers via Passerini reaction. Chem. Commun. 2013, 49 (76), 8549-8551.

33. Li, L.; Deng, X.-X.; Li, Z.-L.; Du, F.-S.; Li, Z.-C., Multifunctional Photodegradable Polymers for Reactive Micropatterns. Macromolecules 2014, 47 (14), 4660-4667.

34. Wilson, D. S.; Dalmasso, G.; Wang, L.; Sitaraman, S. V.; Merlin, D.; Murthy, N., Orally delivered thioketal nanoparticles loaded with TNF- $\alpha-$-siRNA target inflammation and inhibit gene expression in the intestines. Nat. Mater. 2010, 9, 923.

35. Wong, P. T.; Tang, S.; Cannon, J.; Chen, D.; Sun, R.; Lee, J.; Phan, J.; Tao, K.; Sun, K.; Chen, B.; Baker, J. R.; Choi, S. K., Photocontrolled Release of Doxorubicin Conjugated through a Thioacetal Photocage in FolateTargeted Nanodelivery Systems. Bioconjugate Chemistry 2017, 28 (12), 3016-3028.

36. Wong, P. T.; Tang, S.; Cannon, J.; Mukherjee, J.; Isham, D.; Gam, K.; Payne, M.; Yanik, S. A.; Baker Jr., J. R.; Choi, S. K., A Thioacetal Photocage Designed for Dual Release: Application in the Quantitation of Therapeutic Release by Synchronous Reporter Decaging. ChemBioChem 2017, 18 (1), 126-135.

37. Jin, H.; Zhu, T.; Huang, X.; Sun, M.; Li, H.; Zhu, X.; Liu, M.; Xie, Y.; Huang, W.; Yan, D., ROS-responsive nanoparticles based on amphiphilic hyperbranched polyphosphoester for drug delivery: Light-triggered sizereducing and enhanced tumor penetration. Biomaterials 2019, 211, 68-80.

38. Barth, A.; Corrie, J. E.; Gradwell, M. J.; Maeda, Y.; Mäntele, W.; Meier, T.; Trentham, D. R., Time-resolved infrared spectroscopy of intermediates and products from photolysis of 1-(2-nitrophenyl) ethyl phosphates: reaction of the 2-nitrosoacetophenone byproduct with thiols. J. Am. Chem. Soc. 1997, 119 (18), 4149-4159.

39. Shim, M. S.; Xia, Y., A Reactive Oxygen Species (ROS)-Responsive Polymer for Safe, Efficient, and Targeted Gene Delivery in Cancer Cells. Angew. Chem. Int. Ed. 2013, 52 (27), 6926-6929.

40. Böhm, F.; Edge, R.; Lange, L.; Truscott, T. G., Enhanced protection of human cells against ultraviolet light by antioxidant combinations involving dietary carotenoids. J. Photochem. Photobiol. B: Biol. 1998, 44 (3), 211-215.

41. Sivakumar, S.; van Veggel, F. C. J. M.; May, P. S., Near-Infrared (NIR) to Red and Green Up-Conversion Emission from Silica Sol-Gel Thin Films Made with La0.45Yb0.50Er0.05F3 Nanoparticles, Hetero-Looping-Enhanced 
Energy Transfer (Hetero-LEET): A New Up-Conversion Process. J. Am. Chem. Soc. 2007, 129 (3), 620-625.

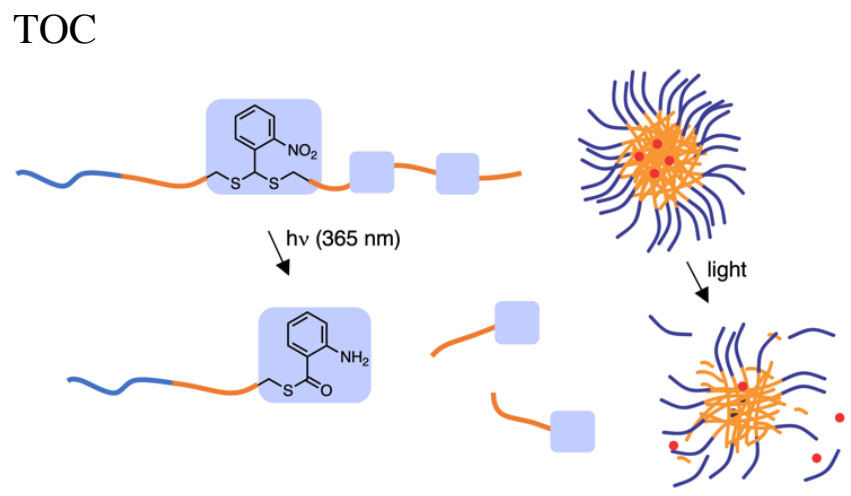

